\title{
Novel lighting properties of white LEDs with two-layered remote phosphor package using red-emitting $\alpha-\mathrm{SrO} \cdot 3 \mathrm{~B}_{2} \mathrm{O}_{3}: \mathrm{Sm}^{2+}$ phosphor
}

\author{
Tran Hoang Quang Minh ${ }^{1}$, Nguyen HuU Khanh Nhan ${ }^{1, *}$, NguYen Doan Quoc AnH $^{2}$, \\ HSIAO-YI LEE ${ }^{1,3}$
}

\author{
${ }^{1}$ Optoelectronics Research Group, Faculty of Electrical and Electronics Engineering, Ton Duc Thang University, \\ Ho Chi Minh City, Vietnam \\ ${ }^{2}$ Faculty of Electrical and Electronics Engineering, Ton Duc Thang University, Ho Chi Minh City, Vietnam \\ ${ }^{3}$ Department of Electrical Engineering, National Kaohsiung University of Applied Sciences, Kaohsiung City, Taiwan
}

\begin{abstract}
This paper investigates a method for improving the lighting performance of white light-emitting diodes (WLEDs), packaged using two separating remote phosphor layers, yellow-emitting YAG:Ce phosphor layer and red-emitting $\alpha-\mathrm{SrO}_{3} \cdot 3 \mathrm{~B}_{2} \mathrm{O}_{3}: \mathrm{Sm}^{2+}$ phosphor layer. The thicknesses of these two layers are $800 \mu \mathrm{m}$ and $200 \mu \mathrm{m}$, respectively. Both of them have been examined at average correlated color temperatures (CCT) of $7700 \mathrm{~K}$ and $8500 \mathrm{~K}$. For this two-layer model, the concentration of red phosphor has been varied from $2 \%$ to $30 \%$ in the upper layer, while in the lower layer the yellow phosphor concentration was kept at $15 \%$. It was found interesting that the lighting properties, such as color rendering index (CRI) and luminous flux, are enhanced significantly, while the color uniformity is maintained at a level relatively close to the level in one-layer configuration (measured at the same correlated color temperature). Besides, the transmitted and reflected light of each phosphor layer have been revised by combining Kubelka-Munk and Mie-Lorenz theories. Through the analysis, it is demonstrated that the packaging configuration of two-layered remote phosphor that contains red-emitting $\alpha-\mathrm{SrO} \cdot 3 \mathrm{~B}_{2} \mathrm{O}_{3}: \mathrm{Sm}^{2+}$ phosphor particles provides a practical solution to general WLEDs lighting.
\end{abstract}

Keywords: remote phosphor structure; two-layered package; luminous flux; color rendering index; light emitting diodes (LEDs)

\section{Introduction}

White light-emitting diodes (WLEDs) that possess high luminous and energy efficiency, small volume, and green environment are good substitutions for conventional incandescent and fluorescent lamps [1]. However, continuous improvements are still needed in light extraction and thermal dissipation from the WLEDs packages $[2,3]$. Blue or UV LEDs combined with phosphor materials can emit white light by converting the generated radiation to a complementary color. In general lighting applications, phosphor-converted LEDs need to have a broadband spectrum which covers the visible region to achieve high color rendering index (CRI). At least, multilayer phosphor

*E-mail: nguyenhuukhanhnhan@tdt.edu.vn packages with different emitting spectra should be selectable. However, due to the electron trapping phenomenon and the inelastic scattering between phosphor materials in LED package, more emission power of LED is lost as the phosphor concentration increases [4]. Phosphor arrangement and geometry have a significant role in enhancing energy efficiency and reducing light trapping in LED packages. Novel phosphor configurations, e.g. scattered photon extraction (SPE) package [5], remote phosphor with a hemispherical dome $[6,7]$ as well as the method to enhance light extraction by using commercial YAG:Ce ${ }^{3+}$ (yellow) and $\mathrm{SrS}: \mathrm{Eu}^{2+}$, $\mathrm{Sr}_{2} \mathrm{Si}_{5} \mathrm{~N}_{8}: \mathrm{Eu}^{2+}$ (red) phosphors $[9,10]$ have been suggested and confirmed by ray-tracing analysis and experiments. Recently, some researchers have also recommended low-concentration phosphor mixture that could provide improvement in 
the output luminous flux and the shift of color coordinates [11]. Other authors proposed an approach to enhance color homogeneity by adding $\mathrm{SiO}_{2}$ [12] or using $\mathrm{Y}_{2} \mathrm{O}_{3}: \mathrm{Eu}^{3+}[8]$. Another method to improve color quality is mixing a red phosphor, like $\mathrm{SrS}: \mathrm{Eu}^{2+}$, with a yellow or green phosphor, like YAG: $\mathrm{Ce}^{3+}$ or $\mathrm{SrGa}_{2} \mathrm{~S}_{4}: \mathrm{Eu}^{2+}$ [13]. These studies are the groundwork for the concept of reducing the backscattering light to LED chip and improving the phosphor converted efficiency [14]. Single-layer, bi-layer or multi-layer structures with a different phosphor concentration at each layer have been reported in the literature $[15,16]$.

Red-emitting $\alpha$-SrO $3 \mathrm{~B}_{2} \mathrm{O}_{3}: \mathrm{Sm}^{2+}$ phosphor, which has a peak wavelength of $680 \mathrm{~nm}$, is a red polycrystalline material. It is synthesized from three oxides including strontium oxide ( $\mathrm{SrO})$, samarium oxide $\mathrm{Sm}_{2} \mathrm{O}_{3}$, and orthoboric acid $\left(\mathrm{H}_{3} \mathrm{BO}_{3}\right) \cdot \mathrm{Sm}^{2+}$ ion is added to the polycrystalline phosphor for enhancing its absorbability at excitation spectrum region of $420 \mathrm{~nm}$ to $502 \mathrm{~nm}$, which results in higher luminous efficiency [17]. Besides, with the advantage of excellent thermal and chemical stability, $\alpha-\mathrm{SrO} \cdot 3 \mathrm{~B}_{2} \mathrm{O}_{3}: \mathrm{Sm}^{2+}$ can be employed for compensating red light, and hence, increasing the color quality of LED lamps. However, until now, there has been no study that employed $\alpha$-SrO $3 \mathrm{~B}_{2} \mathrm{O}_{3}: \mathrm{Sm}^{2+}$ for two-layer RP-WLEDs.

This paper deploys a two-layer remote phosphor structure using a $\alpha-\mathrm{SrO} \cdot 3 \mathrm{~B}_{2} \mathrm{O}_{3}: \mathrm{Sm}^{2+}$ red phosphor layer above a YAG:Ce yellow phosphor layer, and investigates the variance of CRI, CCT deviation and luminous flux that can be caused by adjusting the concentration of red emitting $\alpha-\mathrm{SrO} \cdot 3 \mathrm{~B}_{2} \mathrm{O}_{3}: \mathrm{Sm}^{2+}$ phosphor. The characteristics of the proposed model are compared to one-layer remote phosphor WLED (RP-WLED) package, which has been implemented in a previous study [18]. This investigation has been divided into two tasks: (1) analyzing the effect of $\alpha-\mathrm{SrO} \cdot 3 \mathrm{~B}_{2} \mathrm{O}_{3}: \mathrm{Sm}^{2+}$ concentration on the lighting performance of two-layer RP-WLED by combining Kubelka-Munk and Mie-Lorenz theories; (2) using the LightTools software to simulate the physical models of one-layer and two-layer RP-WLED.

\section{Construction and analysis of physical model}

The LightTools and SolidWorks are necessary to build a remote phosphor multi-chip WLEDs model, which is shown in Fig. 1. The RP-WLED reflector has a bottom length of $8 \mathrm{~mm}$, a height of $2.07 \mathrm{~mm}$ and a length of $9.85 \mathrm{~mm}$ at its top surface. The one-layer remote phosphor configuration, which has a fixed thickness $\mathrm{d}=1 \mathrm{~mm}$, is composed of $\alpha-\mathrm{SrO} \cdot 3 \mathrm{~B}_{2} \mathrm{O}_{3}: \mathrm{Sm}^{2+}$ red phosphor and YAG:Ce yellow phosphor and it is placed at a distance of $0.8 \mathrm{~mm}$ from the 9 LED chips as shown in Fig. 1a. Each chip with a square base of $1.14 \mathrm{~mm}^{2}$ and a height of $0.15 \mathrm{~mm}$ is placed in the cavity of the reflector. The radiant flux of each blue chip is $1.16 \mathrm{~W}$ at a wavelength of $455 \mathrm{~nm}$. Fig. 1b shows a two-layer configuration with $\alpha-\mathrm{SrO} \cdot 3 \mathrm{~B}_{2} \mathrm{O}_{3}: \mathrm{Sm}^{2+}$ red phosphor layer having fixed thickness of $0.2 \mathrm{~mm}$ and YAG:Ce yellow phosphor layer having fixed thickness of $0.8 \mathrm{~mm}$. In order to achieve light output and to investigate the effect of $\alpha-\mathrm{SrO} \cdot 3 \mathrm{~B}_{2} \mathrm{O}_{3}: \mathrm{Sm}^{2+}$ with the same CCT for both types of the studied RP-WLED packages, the amount of red-emitting phosphorsilicone mixture is adjusted while the concentration of yellow-emitting phosphor-silicone mixture is fixed at $15 \%$. Also, the height of encapsulation is kept approximately the same in both configurations. Fig. 1c illustrates the package model and its real sample.

The two-flux model of Kubelka et al. [19] is applied for two-layered structure in the analysis of light properties since it is simple and frequently yields fairly accurate results. We consider two fluxes across the $\mathrm{x}$-direction through a thin layer of thickness $x$ in YAG:Ce yellow phosphor layer of total thickness $d$ as shown in Fig. $1 b$.

The flux $\mathrm{I}_{+}(\mathrm{x})$ in the positive $\mathrm{x}$-direction is decreased by absorption and backscattering inside the layer and is increased by backscattering of the intensity $I_{-}(\mathrm{x})$ in the negative $\mathrm{x}$-direction:

$$
d I_{+}(x)=-(K+S) I_{+}(x) d x+S I_{-}(x) d
$$

In contrast, the flux $I_{-}(x)$ in the negative $\mathrm{x}$-direction is decreased by absorption and 


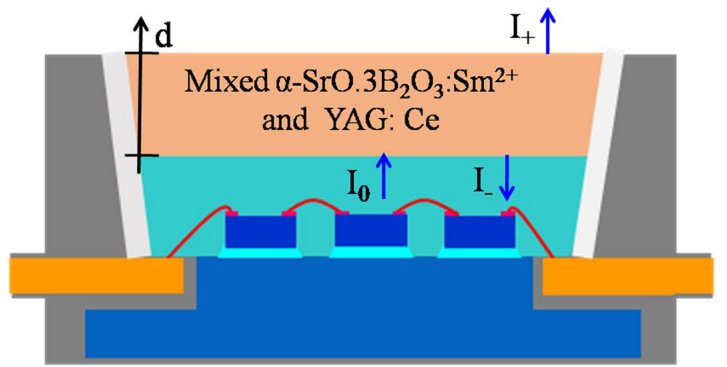

(a)

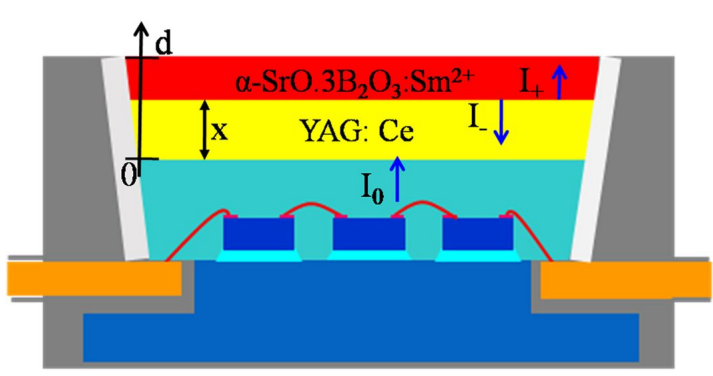

(b)

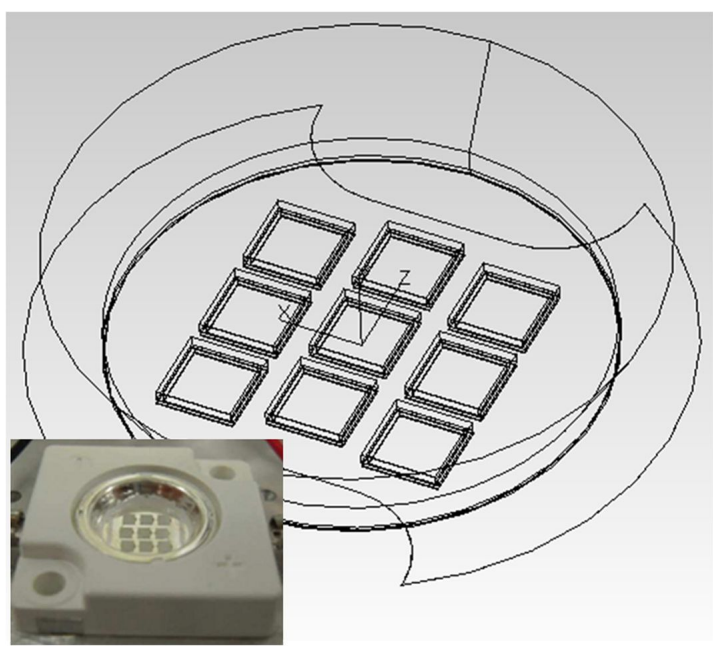

(c)

Fig. 1. Cross-sectional schematic view of the RP-MWLED package: (a) mixed yellow and red phosphor structure; (b) two-layered yellow/red phosphor structure; (c) package model and its real sample.

backscattering inside the layer and is increased by backscattering of the intensity $\mathrm{I}_{+}(\mathrm{x})$ in the positive $\mathrm{x}$-direction:

$$
d I_{-}(x)=-(K+S) I_{-}(x) d x+S I_{+}(x) d x
$$

The above differential equations can be solved only for the ratio $I_{-}(x) / I_{+}(x)$. The results for $x=0$ and $\mathrm{x}=\mathrm{d}$ are of special interests. The ratio $\mathrm{I}_{-}(\mathrm{x}=0) / \mathrm{I}_{+}(\mathrm{x}=0)$ at $\mathrm{x}=0$ defines the diffuse reflectance $\mathrm{R}_{\mathrm{KM}}$ of the two-layered structure:

$$
R_{K M}=\frac{(1+\gamma)(1-\gamma)[\exp (\beta d)-\exp (-\beta d)]}{(1+\gamma)^{2} \exp (\beta d)-(1-\gamma)^{2} \exp (-\beta d)}
$$

Analogously, the ratio $I_{-}(x=d) / I_{+}(x=d)$ at $x=d$ can be used to define the diffuse transmittance $T_{K M}$ of this structure:

$$
T_{K M}=\frac{4 \gamma}{(1+\gamma)^{2} \exp (\beta d)-(1-\gamma)^{2} \exp (-\beta d)}
$$

where $\gamma=\sqrt{\frac{\mathrm{K}}{\mathrm{K}+2 \mathrm{~S}}}$ and $\beta=\sqrt{\mathrm{K}(\mathrm{K}+2 \mathrm{~S})}$. The quantities $\mathrm{K}$ and $\mathrm{S}$ are related to the cross-sections for extinction and scattering, $\mathrm{C}_{\mathrm{ext}}$ and $\mathrm{C}_{\mathrm{sca}}$, the asymmetry parameter $\mathrm{g}$ which presents the amount of back and forward scattered light, and the volume density $V_{d}$ of phosphor particles [20-22]:

$$
\begin{gathered}
K=\frac{C_{e x t}-C_{s c a}}{V_{d}}=\frac{C_{a b s}}{V_{d}} \\
S=\frac{3}{4} \frac{C_{s c a}(1-g)}{V_{d}}=\frac{3}{4} \frac{\delta_{s c a}}{V_{d}}
\end{gathered}
$$

According to Mie-Lorenz theory [23], $\delta_{\text {sca }}$ is the reduced scattering coefficient. The extinction efficiency $\mathrm{C}_{\text {ext }}$, the scattering efficiency $\mathrm{C}_{\text {sca }}$, and the absorption efficiency $\mathrm{C}_{\mathrm{abs}}$ are normally calculated by following equations:

$$
C_{\text {ext }}=\frac{2 p}{k^{2}} 1_{0}^{A_{t}}(2 n+1) \operatorname{Re}\left(a_{n}+b_{n}\right)
$$

$$
C_{s c a}=\frac{2 p}{k^{2}} 1_{0}^{A_{t}}(2 n+1)\left(\left|a_{n}\right|^{2}+\left|b_{n}\right|^{2}\right)
$$

where $\mathrm{k}$ is the wave number $(2 \pi / \lambda)$, and $a_{n}$ and $b_{n}$ are the extension factors with even and odd symmetry, respectively, which are calculated by:

$$
a_{n}(z, m)=\frac{\psi_{n}^{\prime}(m z) \psi_{n}(z)-m \psi_{n}(m z) \psi_{n}^{\prime}(z)}{\psi_{n}^{\prime}(m z) \zeta_{n}(z)-m \psi_{n}(m z) \zeta_{n}^{\prime}(z)}
$$

$$
b_{n}(z, m)=\frac{m \psi_{n}^{\prime}(m z) \psi_{n}(z)-\psi_{n}(m z) \psi_{n}^{\prime}(z)}{m \psi_{n}^{\prime}(m z) \zeta_{n}(z)-\psi_{n}(m z) \zeta_{n}^{\prime}(z)}
$$


where $\psi_{\mathrm{n}}(\mathrm{z})$ and $\zeta_{\mathrm{n}}(\mathrm{z})$ are the Riccati-Bessel functions, $\mathrm{z}$ is relative to the size, which is calculated by equation $11, \mathrm{~m}$ is the refractive index of the particle which contains both the real and virtual term and depends on the ambient medium. In the WLEDs packaging, the ambient medium of phosphor particles is a silicone gel. Thus, the complex refractive index of phosphor particle is calculated as $\mathrm{m}=\mathrm{n}_{\text {phos }} / \mathrm{n}_{\text {sil }}$ :

$$
z=k \cdot r \cdot n_{\text {sil }}=\frac{2 \pi n_{\text {sil }}}{\lambda} \cdot r
$$

where $r$ is the equivalent spherical radius of phosphor particles, $\lambda$ is the wavelength. $n_{\text {phos }}$ and $n_{\text {sil }}$ are the refractive index of phosphor and silicone gel, respectively.

For two-layered structure, the transmission and reflection intensities of blue and yellow light are especially important because the changes in diffuse reflectance $\mathrm{R}_{\mathrm{KM}}$ and transmittance $\mathrm{T}_{\mathrm{KM}}$ also result in the changes of optical properties of WLEDs. In Fig. 2 and Fig. 3, we can observe the interaction among the light scattering, reduced scattering, backscattering, and conversion inside the two phosphor layers. These results have been obtained by theoretical calculations with $\alpha-\mathrm{SrO} \cdot 3 \mathrm{~B}_{2} \mathrm{O}_{3}: \mathrm{Sm}^{2+}$ concentration varying from $2 \%$ to $35 \%$, whereas YAG:Ce yellow phosphor concentration has been fixed at $15 \%$. The average radius of the two types of phosphor configurations has been assumed as $7.25 \mu \mathrm{m}$. The refractive indexes of silicone glue, $\alpha-\mathrm{SrO} \cdot 3 \mathrm{~B}_{2} \mathrm{O}_{3}: \mathrm{Sm}^{2+}$ and YAG:Ce particles have been chosen as 1.5, 1.93, and 1.83, respectively [12].

In Fig. 2a, it can be seen that the scattering and reduced scattering coefficients show a rapidly rising trend for blue light $(\lambda=455 \mathrm{~nm})$, yellow light $(\lambda=595 \mathrm{~nm})$ and red light $(\lambda=680 \mathrm{~nm})$. Moreover, the scattering coefficient and reduced scattering coefficient of blue light increase more than both yellow and red light in the yellow phosphor layer. Meanwhile, scattering coefficient of blue light in the red phosphor layer also gets greater than that of yellow light, but the reduced scattering coefficient is not much different (Fig. 2b). Backscattering coefficient at the wavelength of $455 \mathrm{~nm}$

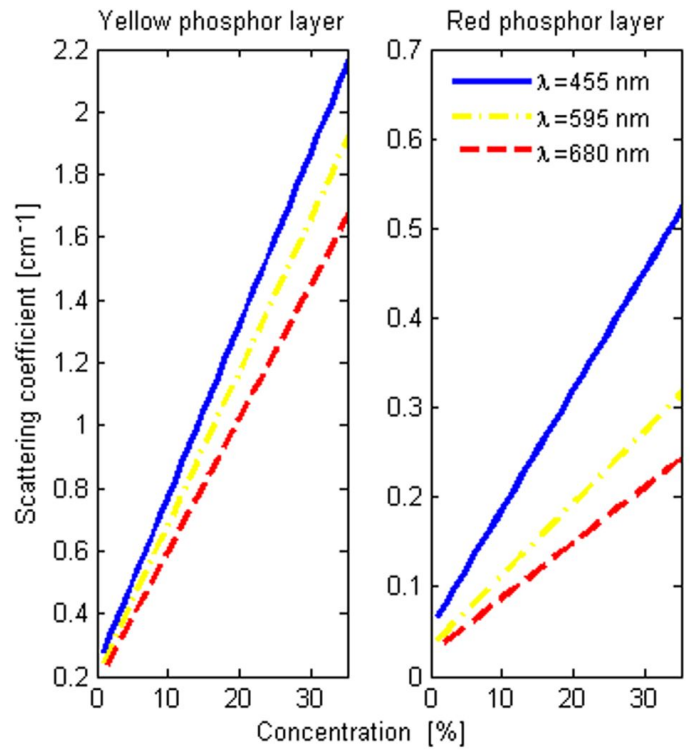

(a)

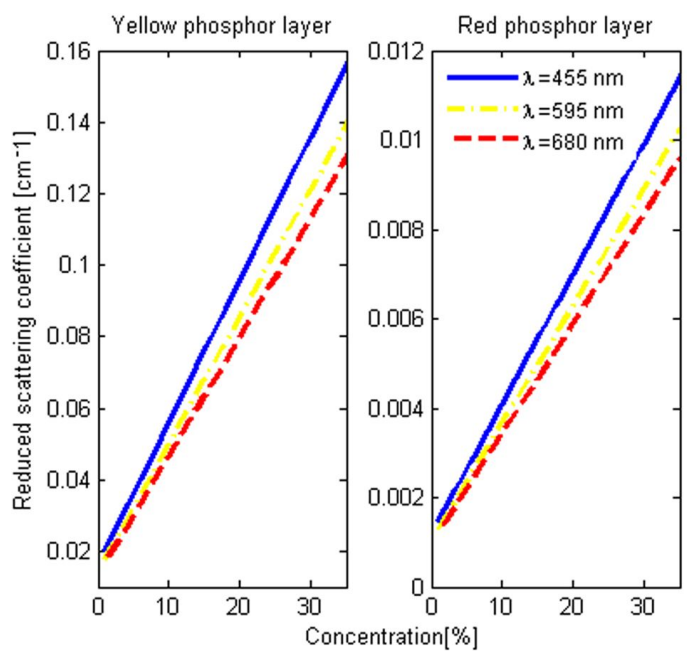

(b)

Fig. 2. The effect of $\alpha-\mathrm{SrO} \cdot 3 \mathrm{~B}_{2} \mathrm{O}_{3}: \mathrm{Sm}^{2+}$ concentration in two-layered red/yellow phosphor structure on (a) scattering coefficient and (b) reduced scattering coefficient at wavelengths of $455 \mathrm{~nm}$, $595 \mathrm{~nm}$ and $680 \mathrm{~nm}$.

is much larger than at the wavelength of $595 \mathrm{~nm}$ and $680 \mathrm{~nm}$ in yellow phosphor layer as shown in Fig. 3. For both blue and yellow lights in the red-emitting phosphor layer, the backscattering coefficient is much lower than the one at $680 \mathrm{~nm}$ (red) light. These phenomena may be caused by the following reasons: (1) When $\alpha-\mathrm{SrO} \cdot 3 \mathrm{~B}_{2} \mathrm{O}_{3}: \mathrm{Sm}^{2+}$ 
concentration rises, more blue, yellow and red light are absorbed and scattered. It becomes harder for blue and yellow light to penetrate the phosphor layer. Thus, the light intensity changes extraordinarily, which implies a continuous variability of transmitted and reflected light in Fig. 4. (2) With the increasing of $\alpha-\mathrm{SrO} \cdot 3 \mathrm{~B}_{2} \mathrm{O}_{3}: \mathrm{Sm}^{2+}$ concentration, blue light is further backscattered in all directions in both yellow and red phosphor layers. Thus, the reflected blue light has a tendency to increase. The further increasing of $\alpha-\mathrm{SrO} \cdot 3 \mathrm{~B}_{2} \mathrm{O}_{3}: \mathrm{Sm}^{2+}$ concentration does not account for the rising of intensity at the incident surface, so the transmitted light intensity tends to decrease in Fig. 4. (3) When red phosphor concentration increases, at first more yellow light is converted.

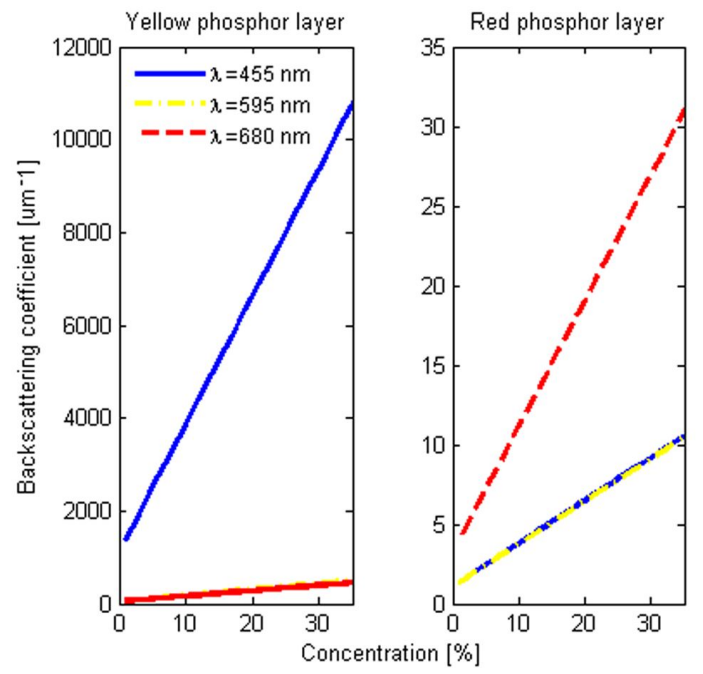

Fig. 3. Backscattering coefficient of two-layered phosphor structure at wavelengths of $455 \mathrm{~nm}, 595 \mathrm{~nm}$ and $680 \mathrm{~nm}$.

When the concentration increases further, the effect of yellow light absorption and backscattering is the same as that of blue light, which explains the increasing in transmitted light intensity at the wavelength of $595 \mathrm{~nm}$ (shown in Fig. 4). This increase leads to a wider emitted spectrum at the wavelength close to $595 \mathrm{~nm}$, similar to the theoretical calculation spectra for two-layered LED in Fig. 5, or the changes in diffuse reflectance and transmittance which also result in the color index variation of the WLEDs.

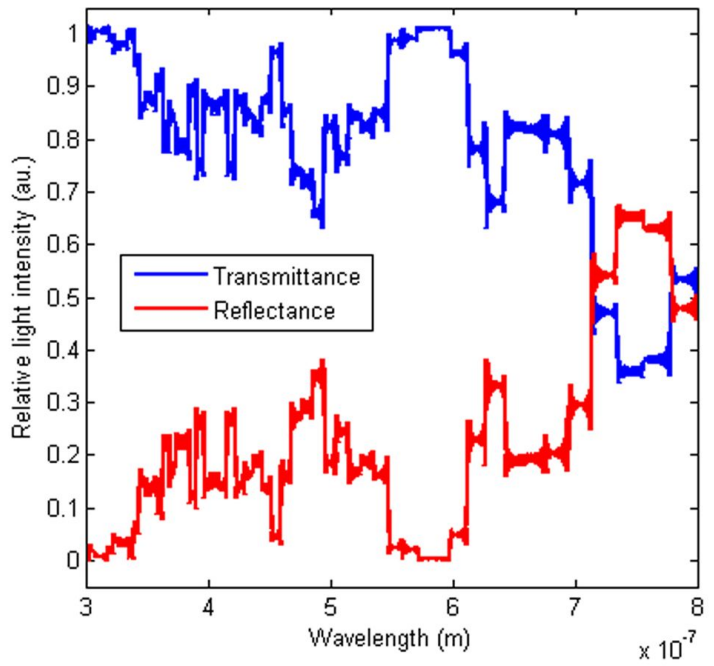

Fig. 4. Computed diffuse reflectance and transmittance spectra of two-layered phosphor structure.

\section{Results and discussion}

The emission spectra of two-layer and mixed one-layer WLED have been obtained by running optical ray-tracing of LightTools ${ }^{\mathrm{TM}}$ software at the same correlated color temperature (CCT) of $8500 \mathrm{~K}$. The experimental results have been compared to the theoretical values at the same concentrations of yellow and red phosphor particles, using Matlab software, as shown in Fig. 5. The emission spectra of the blue, yellow and red phosphors with the blue LED excitation light source are illustrated in Fig. 5a. These spectral power distributions have been measured using optical simulation. Both YAG:Ce and $\alpha-\mathrm{SrO} \cdot 3 \mathrm{~B}_{2} \mathrm{O}_{3}: \mathrm{Sm}^{2+}$ phosphors have been simulated based on the extended Gaussian model [25] described by equation 13 . For multiple phosphor layers (yellow and red), converted yellow radiant power from the first layer is divided into $\mathrm{Y}_{1}$ and $\mathrm{Y}_{2}$. While $\mathrm{Y}_{1}$ could be absorbed by the red phosphor because it lies within the absorption (extinction: $\mathrm{C}_{\mathrm{ext}}$ ) spectral range of red phosphor, $\mathrm{Y}_{2}$ cannot be absorbed by the red phosphor (Fig. 5a). The reflected and transmitted light would include some portion of the blue, red, and yellow $\left(\mathrm{Y}_{1}\right.$ and $\mathrm{Y}_{2}$ ) radiations for blue radiant power incident on multiple phosphor layers. Therefore, in the total spectral power distribution modeling for 


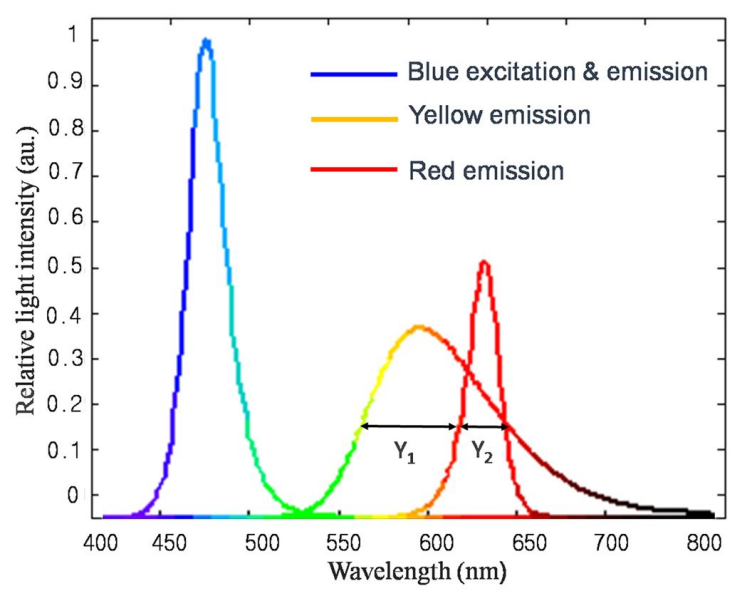

(a)

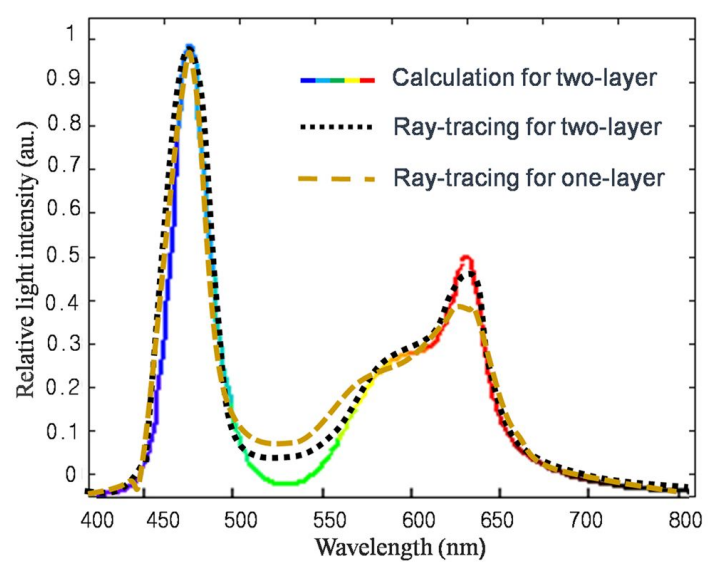

(b)

Fig. 5. (a) Emission spectra of the blue, yellow and red phosphors with the blue LED excitation light source; (b) theoretical calculation of the light distribution spectra vs. optical ray-tracing in the remote phosphor package at $8500 \mathrm{~K}$.

two-layer-coated phosphors, white LED can be expressed as a tricolor spectrum (B-Y-R) (Fig. 5b).

The optical ray-tracing results match well to the theoretical calculations in two-layered structure. The spectral energy distributions in the WLEDs emission light and color features, in this case, are determined by a constitution of the relative luminous energy of the converted photons in phosphor layers. The reason for this disagreement results from the one-layer LED structure, where a considerable percentage of the translated photons involved in backscattering are absorbed (flux I(x)) by the multichip LED. However, in two-layer structure, these backscattered photons are taken out of the LED package, in addition to the total energy spectrum. Furthermore, the forward-emitted (flux $\mathrm{I}_{+}(\mathrm{x})$ ) intensity from the first (YAG:Ce) layer will go through the second $\left(\alpha-\mathrm{SrO} \cdot 3 \mathrm{~B}_{2} \mathrm{O}_{3}: \mathrm{Sm}^{2+}\right)$ phosphor layer and experience a great scattering changes (Fig. 2b), while the second phosphor layer can absorb significant photon quantity which declines in the excited energy band. However, the two-layer configuration provides a higher percentage of photons to the total light energy than a single phosphor layer. For that reason, the first phosphor layer turns out the forceful layer. This causes that the output luminous flux of two-layer LED is at least $17 \%$ higher than that of mixed onelayer LED. It has been demonstrated that highconcentration of red phosphor $\alpha-\mathrm{SrO} \cdot 3 \mathrm{~B}_{2} \mathrm{O}_{3}: \mathrm{Sm}^{2+}$ that exceeds $20 \%$ is disadvantageous for the output luminous flux as shown in Fig. 6, due to the light scattering and absorption between phosphors materials as mentioned above.

The inelastic scattering of light reduces the efficiency since the increased light path length leads to higher possibility of re-absorption of the incident and emitted light. Light path randomization also increases the probability of light incident on high loss areas. Compared to the mixed one-layer LED at the same CCT of $7700 \mathrm{~K}$ and $8500 \mathrm{~K}$, the increased radiation flux of blue, yellow and red components in two-layer LED indicate the less scattering of light inside the package. To provide further proof that the total emission power $\mathrm{T}_{\mathrm{RP}}$, including transmittance and reflectance, can be described in the spectral formula depending on wavelength $\lambda$ and phosphor particles radius $r$, from equation 3 to equation 11 as:

$$
T_{R P}(\lambda)=T_{K M}(\lambda)+R_{K M}(\lambda)
$$

From equation 12, the luminous flux (lumen) can be calculated as [24]:

$$
\Gamma=\Psi_{m} \sum T_{R P}(\lambda) \eta(\lambda) \delta(\lambda)
$$

where $\Psi_{m}$ is a constant, $\eta(\lambda)$ is the relative photonic luminous efficiency function, and $\delta(\lambda)$ is the wavelength interval. 
The WLED color properties also depend on the spectral power distribution, which depends on the relative radiant power of the phosphor-converted photons in both layers. Fig. 7 shows the plots of CRI of two-layer and mixed one-layer WLEDs with various red phosphor concentrations at correlated color temperature (CCT) $7700 \mathrm{~K}$ and $8500 \mathrm{~K}$. Theoretical calculation and ray-tracing simulation spectra agree well. The CRIs values are quite different for the two phosphor configurations, although the amounts of yellow and red phosphors are equal in all cases. The two-layered package, which supports longer-wavelength of red phosphor than the single-layer one, yields about $5 \%$ higher CRI output. With the increment of red phosphor concentration, the CRI moves up, to the same extent, in both two-layer and mixed one-layer LEDs under CCT modes. This indicates that their scattering and illumination characteristics are the same; however, for two-layered LED packages the chromaticity of LED can be efficiently adjusted by the ratio of blue to yellow and red components since the yellow phosphor does not absorb the backscattering of red emission from the upper red phosphor layer. This confirms that the emission energy loss is associated with the re-absorption process by different phosphor emission spectra within the LED packages.

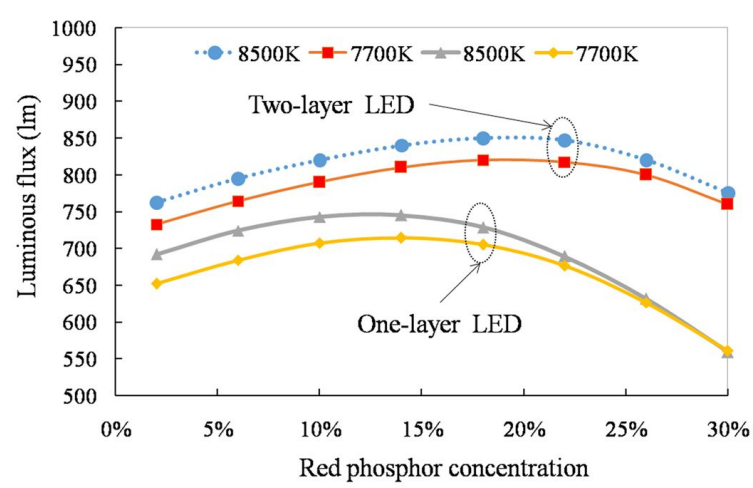

Fig. 6. Luminous flux of two-layer and mixed one-layer LEDs with various red phosphor concentrations.

Another improvement of the CRI is to overcome the derating of light sources at very low and very high CCTs below $3500 \mathrm{~K}$ and above $6500 \mathrm{~K}$, respectively. Different CCT deviations

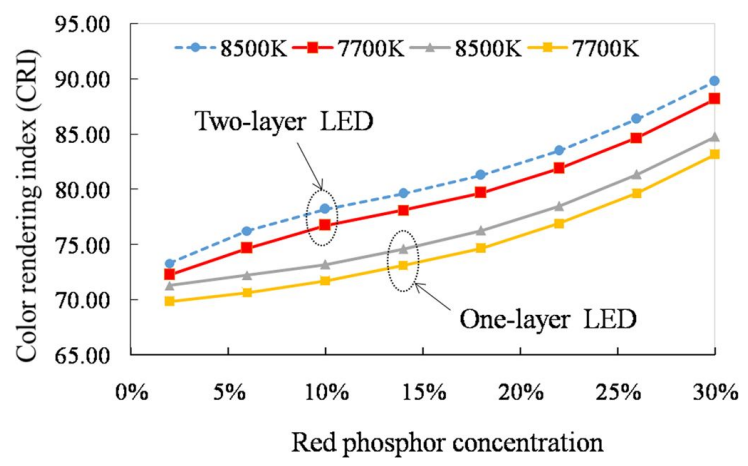

Fig. 7. CRI of two-layer and mixed one-layer LED with various red phosphor concentrations.

could be required in various typical standards for WLED applications. The higher CCT deviation would lead to non-uniform white color at different angles because the light passes through the phosphor layer with higher concentration to get a smaller CCT deviation. As a result, the different lights could be mixed perfectly to form the uniform white light. That is the reason why CCT deviation in one-layered LED package decreases a little with the increment of $\alpha-\mathrm{SrO} .3 \mathrm{~B}_{2} \mathrm{O}_{3}: \mathrm{Sm}^{2+}$ concentration percentage as shown in Fig. 8. Meanwhile, the CCT deviation in two-layered LED package, where the yellow phosphor layer concentration is fixed at $15 \%$ and $\alpha-\mathrm{SrO} .3 \mathrm{~B}_{2} \mathrm{O}_{3}: \mathrm{Sm}^{2+}$ concentration rises from $2 \%$ to $15 \%$, changes insignificantly. However, at red phosphor concentration higher than $15 \%$, CCT deviation tends to increase significantly due to scattering and backscattering in red phosphor layer with $\alpha-\mathrm{SrO}_{3} 3 \mathrm{~B}_{2} \mathrm{O}_{3}: \mathrm{Sm}^{2+}$ particles raised up as in Fig. 2a and Fig. 3. As a result, non-uniform WLED color is generated at different angles. This results in different spectral power distributions of light from two different phosphor configurations, such as in Fig. 5.

\section{Conclusions}

Remote phosphor multi-chip WLEDs with separating $\alpha-\mathrm{SrO} \cdot 3 \mathrm{~B}_{2} \mathrm{O}_{3}: \mathrm{Sm}^{2+}$ (red) and YAG:Ce (yellow) phosphor layers are studied at different $\alpha-\mathrm{SrO} \cdot 3 \mathrm{~B}_{2} \mathrm{O}_{3}: \mathrm{Sm}^{2+}$ concentrations. It is found that this new packaging method with red-emitting $\alpha-\mathrm{SrO} \cdot 3 \mathrm{~B}_{2} \mathrm{O}_{3}: \mathrm{Sm}^{2+}$ phosphor leads to at least 


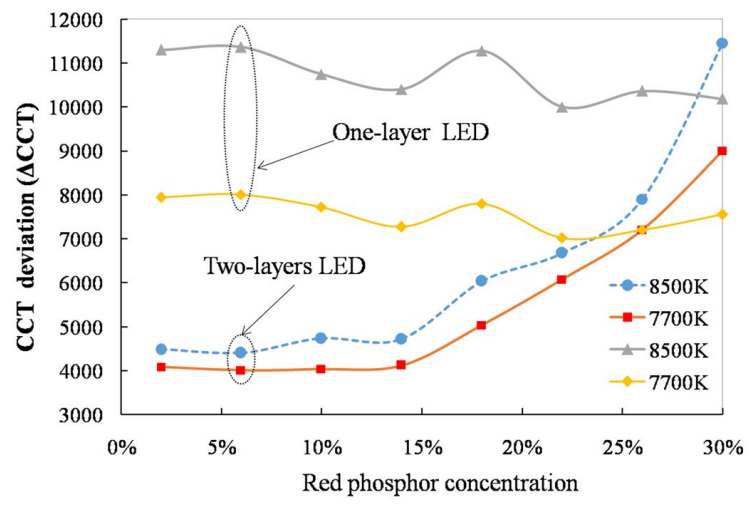

Fig. 8. CCT deviation of two-layer and mixed one-layer LED with various red phosphor concentrations.

more than $17 \%$ increase in lumen compared to traditional mixed red and a yellow phosphor packaging at the same correlated color temperature. The effect of increased concentration $\alpha$-SrO-3B $\mathrm{B}_{2} \mathrm{O}_{3}: \mathrm{Sm}^{2+}$ in two-layered RP-WLEDs is also observed. This improvement in uniform color properties is due to increased scattering and back reflection of light inside the packages. The CRI of two-layered remote phosphor white LEDs is almost $5 \%$ higher than that of mixed phosphor white LEDs in the stable condition of $\alpha$-SrO. $3 \mathrm{~B}_{2} \mathrm{O}_{3}: \mathrm{Sm}^{2+}$ concentration. The technology of two-layered remote phosphor multi-chip WLED package that employs $\alpha-\mathrm{SrO} \cdot 3 \mathrm{~B}_{2} \mathrm{O}_{3}: \mathrm{Sm}^{2+}$ phosphor can give high lumen and color rendering index and allowable uniform color range. These results offer a prospective practical solution to manufacturing two-layered remote phosphor WLEDs. In further works, the influence of $\alpha-\mathrm{SrO} \cdot 3 \mathrm{~B}_{2} \mathrm{O}_{3}: \mathrm{Sm}^{2+}$ phosphor features on the lighting performance of RP-WLEDs will be studied.

\section{References}

[1] Pin-Chao W., Yan-Kuin S., Chun-Liang L., GUAN-SYUN H., IEEE Electr. Device L., 6 (2014), 657.

[2] Arik M., Becker C., Weaver S., Petroski J., Proc. SPIE 5187, 64 (2004), 64.

[3] Mueller-Mach R., Mueller G.O., Krames M.R., Trottier T., IEEE J. Sel. Top. Quant., 2 (2002), 339.
[4] You J.P., Tran N.T., Lin Y.C., He Y., Shi F.G., $J$. Electron. Mater., 6 (2009), 761.

[5] NAREndran N., Gu Y., FreysSinier-Nova J.P., ZHU Y., Phys. Status Solidi. A, 6 (2005), R60.

[6] LuO H., Kim J.K., SChubert E.F., ChO J., Sone C., PARK Y., Appl. Phys. Lett., 24 (2005), 243505.

[7] Kim J.K., Luo H., Schubert E.F., Cho J., Sone C., PArk Y., Jpn. J. Appl. Phys., 21 (2005), L649.

[8] Seong, K.K., Yoo T.W., Kim B.-S., LeE S.M., LeE Y.S., PARK L.S., Mol. Cryst. Liq. Cryst., 1 (2012), 33,

[9] Allen S.C., SteCKL A.J., J. Disp. Technol., 2 (2007), 155.

[10] Allen S.C., Steckl A.J., J. Appl. Phys., 92 (2008), 143309.

[11] Masui H., Nakamura S., Denbaars S.P., Jpn. J. Appl. Phys., 34 (2006), L910.

[12] QUOC A.N.D., LaI M.F., MA H.Y., LEE H.Y., J. Chin. Inst. Eng., 38 (2015), 1354.

[13] Mueller-Mach R., Mueller G., Krames M.R., Hoppe H.A., StAdLER F., SCHNiCK W., JuEstel T., Schmidt P., Phys. Status Solidi. A, 9 (2005), 1727.

[14] Run H., Bin C., Yong Z., Yongming Z., ХiaobING L., IEEE Photonic. Tech. L., 12, (2013), 156.

[15] Chung H.C., Syuan J.G., Ting S.Z., Sheng Y.C., IEEE Electr. Device L., 7 (2016), 898.

[16] Zhu Y., Narendran N., Jpn. J. Appl. Phys., 10R (2010), 100203.

[17] Yen W.M., Weber M.J., Inorganic Phosphors: Compositions, Preparation and Optical Properties, CRC Press, Washington D.C., 2004.

[18] Minh Q.H. Tran, Nhan K.H. NguYen, QuOC ANH D.N., J. Chin. Inst. Eng., 4 (2016), 313.

[19] Kubelka P., Munk F., Z. Tech. Phys., 12 (1931), 593.

[20] Mudgett P.S., Richards L.W., Appl. Optics, 10 (1971), 1485.

[21] Brinkworth B.J., Appl. Optics, 1 (1972), 1434.

[22] ReIss H., Radiative Transfer in Nontransparent Dispersed Media, Springer, Berlin, 1988.

[23] Zhong J., Xie M., Ou Z., Zhang R., Huang M., ZhaO F., Proceedings Symposium on Photonics and Optoelectronics (SOPO), Wuhan, 2011.

[24] Wyszecki G., Stiles W.S., Color Science - Concepts and Methods, Quantitative Data and Formulae, Wiley, New York, 1982.

[25] Huanting C., Hui S.Y., IEEE T. Ind. Electron., 2 (2014), 784 